\title{
Open to interpretation
}

One hundred years after the birth of quantum theory, we are still arguing over what it means. No one disputes the mathematical machinery and how to use it, but nearly everyone has their own private theory about what really goes on behind the scenes (if anything). These assertions, it must be said, seemingly never lead to any testable predictions.

As a student I was, like most of us, indoctrinated into the BohrCopenhagen position: it is simply impossible to picture what goes on in the quantum world. Hence my surprise (and delight) one day when flicking through a 1952 volume of the Physical Review to see that David Bohm had achieved that very thing. A figure in his paper, A Suggested Interpretation of the Quantum Theory in Terms of 'Hidden' Variables, illustrates how particles, according to this particular scheme, can move through a two-slit apparatus on definite trajectories, yet still produce an interference pattern. Apparently, it wasn't as inconceivable as Bohr had said.

Bohm deserves credit, along with John Bell later, for making it possible once again to explore heretical ideas about quantum mechanics.
Today, there are at least seven different 'interpretations' of quantum theory on the go (and with at least some claim to consistency). Aside from Bohm's theory, there's the Many Worlds idea, the Consistent Histories approach, the Transactional Interpretation and theories of 'spontaneous collapse', as well as the old-style Copenhagen Interpretation and David Mermin's version, the Ithaca Interpretation, named for the location of Cornell University.

Behind this lies the problem that quantum superpositions exist at the level of individual particles or small numbers of particles, yet no one has ever seen it at the macro-level (unless you count relatively small magnetic clusters as 'macro'). This is a serious issue for anyone who still expects physics to find a seamless theoretical description of reality, rather than a patchwork of incompatible theories restricted to their own domains.

In sewing the world back together, most people seem to agree on the likely importance of the environment. Ironically, this point was made as long ago as 1950, also by Bohm, in his textbook on quantum theory, in a chapter on the measurement process. This idea - now evolved into the theory of decoherence - is,

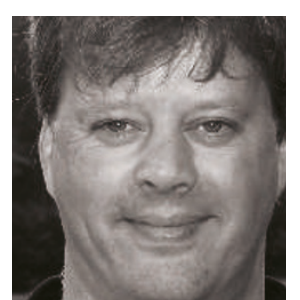

in essence, quite simple. Measurement entangles a quantum system with a macroscopic object having an astronomical number of degrees of freedom, almost all of which remain outside the experimenter's control. If you average over these inaccessible variables, as all present experiments do, the theoretical description of the quantum system becomes a diagonal density matrix representing a mixed state. The system acts as if any superpositions had collapsed.

It's hard to believe that this isn't a part of the real solution to the problem. Even so, as Bell pointed out, it offers at best a FAPP solution ('for all practical purposes'), not a fundamental one. If the linear evolution of quantum theory is correct, then a world of historical information still resides in the state vector of the environment; it doesn't have to be averaged over, and could, in principle, come back to have later consequences.

Some theorists have been exploring the meaning of this 'hidden' information. Does it have measurable consequences, or not? One day, it would be nice to think, one of these interpretations will indeed have some detectable consequences.

Mark Buchanan

\section{Spring fever}

Perhaps it is just the onset of spring, but I am feeling optimistic.

The team behind the WMAP Cosmic Microwave Background satellite has just released its thirdyear data, which remain strikingly consistent with many of the canonical predictions of inflationary theories (despite some puzzling features at large angular scales).

Next year, the new Large Hadron Collider at CERN will be turned on. We have been waiting over 20 years for an accelerator capable of definitively addressing physics beyond the standard model, thanks to the tragically stupid cancellation of the Superconducting Supercollider project in the United States - which would otherwise by now have been running for almost a decade.
And in the last year, the Laser Interferometer Gravitational-Wave Observatory (LIGO) has reached its design sensitivity, something many in the community thought unachievable. Although LIGO-1 is unlikely to detect gravitational waves from astrophysical sources, engineering lessons of the past decade enabled improvements through Advanced LIGO and ultimately to space-based interferometers. These might reveal precise information not only on the final stages of blackhole collapse, but on the expansion history of the Universe.

We are not guaranteed any revolutionary discoveries, but if history is any guide, every time we open new windows on the Universe we tend to be surprised. Even null experiments, such as that performed

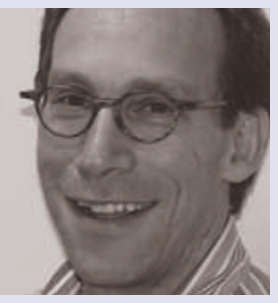

A LONG WINTER'S DARKNESS MAY SOON BE COMING TO AN END. almost 120 years ago by Albert Michelson and Edward Morley, can be profoundly important.

This will be especially important to remember if the LHC reveals nothing new. Although it may make funding the proposed International Linear Collider more difficult, whether we observe the standard-model Higgs, or don't observe the Higgs at all, this will dramatically alter the theoretical playing field of fundamental physics. Notions such as lowenergy supersymmetry - perhaps the most well-motivated, untested theory on the market - may need to be revised or discarded.

One way or another, a long winter's darkness may soon be coming to an end.

Lawrence M. Krauss 